\title{
Integrating Carbon Nanotubes For Atomic Force Microscopy Imaging Applications
}

Qi Ye ${ }^{*, 1,2}$, Alan M. Cassell ${ }^{1,2}$, Hongbing Liu², Jie Han ${ }^{1,2}$, and Meyya Meyyappan ${ }^{1}$

${ }^{1}$ Center for Nanotechnology, NASA Ames Research Center, Moffett Field, California 94035, U.S.A.

${ }^{2}$ Integrated Nanosystems, Inc., NASA Research Park, Moffett Field, California 94035, U.S.A.

*Corresponding author: qye@mail.arc.nasa.gov

\begin{abstract}
Carbon nanotube (CNT) related nanostructures possess remarkable electrical, mechanical, and thermal properties. To produce these nanostructures for real world applications, a large-scale controlled growth of carbon nanotubes is crucial for the integration and fabrication of nanodevices and nanosensors. We have taken the approach of integrating nanopatterning and nanomaterials synthesis with traditional silicon microfabrication techniques. This integration requires a catalyst or nanomaterial protection scheme. In this paper, we report our recent work on fabricating wafer-scale carbon nanotube AFM cantilever probe tips. We will address the design and fabrication considerations in detail, and present the preliminary scanning probe test results. This work may serve as an example of rational design, fabrication, and integration of nanomaterials for advanced nanodevice and nanosensor applications.
\end{abstract}

\section{INTRODUCTION}

Carbon nanotubes (CNTs) have been widely studied in recent years [1]. Due to their remarkable electrical, mechanical, and thermal properties, CNTs become very attractive in a wide variety of applications in sensors, microelectronics, field emitters, and nanodevices, etc. [15]. However, a great challenge of CNTs in their applications relies on the integration of these nano-scale materials with micron scale structures to form workable devices and sensors.

Although there are numerous scientific papers revealing the structures and properties of these nanomaterials, not much research and development work have been conducted to address the issue of nanomaterials integration for nanodevice and nanosensor fabrications and applications. To move nanoscience and nanotechnology forward, we aim to develop an innovative methodology for the reliable integration of nanoscale materials with CMOS microfabrication technologies for making nanodevices and nanosensors at wafer-scale. Our methodology includes four major steps: (1) Catalyst nanopatterning at wafer-scale; (2) Integration of nanopatterning with traditional microfabrication of silicon devices; (3) Reliable electric field enhanced chemical vapor deposition for vertical CNT. growth; and (4) CNT-based devices performance evaluation and testing. In this paper, we describe a detailed wafer-scale fabrication process for making CNT AFM probe tips that are used for tapping-mode imaging applications.

\section{EXPERIMENTAL DETAILS}

\section{(1) Catalyst nanopatterning at wafer-scale}

In order to solve the problems that exist with conventional CNT based imaging probes, we have developed a scanning probe in which the CNT tip is directly grown from the silicon 
cantilever, not manually attached or randomly grown. In developing this solution, a key hurdle is to integrate CNT growth with traditional silicon cantilever fabrication so that reliable catalyst nanopatterning and registration are accomplished at the wafer scale. The patterned catalyst dot sizes are typically in the range of 75 to $300 \mathrm{~nm}$ and the process layer-by-layer alignment accuracies are in the range of $t /-1 \mu \mathrm{m}$. These are quite challenging requirements for nanopatterning at the wafer scale. E-beam lithography is used in this study for patterning various catalyst feature sizes from $75 \mathrm{~nm}$ to $300 \mathrm{~nm}$. A highly sensitive bilayer resist composed of low and high molecular weight poly(methylmethacrylate) (PMMA) (100 nm total PMMA thickness) was applied to the wafer, and then exposed at varied beam dosage conditions using the highspeed electron beam writer Hitachi HL-700F e-beam system. After nanopatterning, the resist layer was developed. $20 \mathrm{~nm} \mathrm{Cr}$ and $20 \mathrm{~nm} \mathrm{Ni}$ (catalyst layers) were deposited using electron beam evaporation. The resist was then lifted-off, leaving only the $\mathrm{Cr} / \mathrm{Ni}$ catalysts at the defined locations on wafer.

\section{(2) Integration of nanopatterning with traditional microfabrication of silicon cantilevers}

In order for the catalyst seeds that define the CNT locations to survive harsh chemical etchants (such as $\mathrm{KOH}$ ) required for cantilever fabrication, we developed catalyst protection schemes before commencing the conventional microfabrication process. Two protection schemes have been tested: (i) nitride layer protection, and (ii) specialty chemical etch protective coating protection. One set of wafers was deposited with $200 \mathrm{~nm}$ thick $\mathrm{Si}_{3} \mathrm{~N}_{4}$ layer. The other set of wafers was primed first with ProTEK EXP03062-B and then spin-coated with ProTEK EXP02103-18 etch protectant (both ProTEK chemicals were obtained from Brewer Science, Inc.). The wafers were then subjected to a series of photolithographic patterning, dry and wet etching process steps to fabricate the cantilevers from SOI wafers $(10 \mu \mathrm{m}$ device layer, $1 \mu \mathrm{m}$ oxide layer and $380 \mu \mathrm{m}$ handle layer).

The cantilevers were made from a two-mask fabrication process. The front-side mask gave the outline of the cantilevers (cantilever body, cantilever beam, and the tabs that hold the cantilever to the silicon wafer frame). The backside mask defined the body of the cantilevers. Each fabrication step was aligned to the global alignment marks that were patterned at the ebeam lithographic step. This insured that the catalyst sites and the final CNTs were located at the exact locations on the cantilever beam where they were designed. Based on the $\mathrm{x}$ and $\mathrm{y}$ coordinates and the expected stitching accuracy of the electron beam writer (accuracy is better than $0.5 \mu \mathrm{m}$ ), we have chosen to place the catalyst spot at the center of the cantilever beam and 5 $\mu \mathrm{m}$ away from the cantilever diving board end. A total of 244 cantilevers were made from a $4 "$ SOI wafer. After microfabrication was completed, the protection layers were stripped, leaving the catalyst sites exposed. We then proceeded with wafer-scale CNT growth.

\section{(3) Reliable electric field enhanced chemical vapor deposition for vertical CNT growth}

We designed and built a 6" hot-filament DC plasma enhanced chemical vapor deposition (PECVD) reactor. Our CNT growth was achieved at 4" wafer scale. This reactor permits vast flexibility in process parameter control and monitoring. Through our innovative design, we can vary the normal process variables such as pressure, plasma power, and gas mixture flow and composition as well as a number of additional process variables such as electrode gap, electrode geometry (for angled CNT growth), substrate temperature, and hot filament heating. In addition, 
the reactor is equipped with a residual gas analyzer (RGA), which permits us to monitor the relevant gas phase intermediates important to our growth process [6]. After a systematic approach, we were able to obtain individual CNTs from various catalyst sizes on cantilevers. Scanning electron microscopy was used to characterize the catalyst feature sizes prior to and after growth for comparison. To assess the crystalline quality of the individual CNTs on cantilevers after growth, we performed cross-sectional transmission electron microscopy analysis using our specially designed chip holder that allowed for direct inspection of the as-grown CNT tips. The high quality CNT probes were then mounted into an AFM for probe mechanical strength and imaging capability tests.

\section{(4) CNT AFM probe tip performance evaluation and testing}

It is critical to validate the usefulness of the CNT AFM probe tips. Our characterization focused on mechanical stability of the CNT probe tips as they engaged the imaging surface in a conventional AFM operation under tapping mode acquisition. We also performed CNT probe imaging lifetime study by continuously scanning CNT probes for many hours in ambient environment. We studied CNT probe imaging resolution by scanning the probe over an array of $2 \mu \mathrm{m}$ circular holes that were $300 \mathrm{~nm}$ deep. The images from all these studies were compared with traditional silicon AFM tips in parallel. We also studied the interaction curves derived from tapping mode imaging (amplitude and deflection signals) to characterize the buckling signature that was characteristic of CNTs.

\section{DISCUSSION}

While electron beam lithography is generally considered as an academic research tool, and is slow and expensive for most CMOS manufacturing processes, it only takes us about 20 minutes write time to pattern the limited number of nanometer scale dots ( 244 in this case) on a 4 " wafer. Compared to other nanopatterning techniques (deep or extreme Ultraviolet lithography, nanoimprinting, etc.), e-beam lithography has given us the best results in regards to wafer-scale overall alignment accuracy, dot size uniformity, reliability, yields, and process economics. The dot size can be easily tailored by redesigning a new e-beam pattern file, while for other nanopatterning methods, expensive masks or molds need to be re-made every time a single dot size needs to be varied. E-beam lithography also provides the least overall stitching problem for nanopatterning over a 4" wafer area. It is very simple to make and register the global and local alignment marks via the same lithographic step. Therefore, the process integration of nano- and microfabrication is feasible.

With the appropriate protection scheme, nano-catalysts survive the cantilever microfabrication process without any problems. For the two protection schemes that we investigated, we have found that nitride layer protection worked the best for dry etching process, while ProTEK EXP chemicals worked the best for wet etching ( $\mathrm{KOH}$ etching).

A formidable challenge for the development of CNT probe tips is the controlled growth of individual CNTs possessing the desired properties (crystallinity, diameter, length, and orientation) and placement on silicon cantilever substrates. Using the optimized catalyst formulation $(\mathrm{Cr} / \mathrm{Ni})$ and process gas mixture $\left(80 \mathrm{sccm} \mathrm{NH} \mathrm{NH}_{3}\right.$ and $22.5 \mathrm{sccm} \mathrm{C}_{2} \mathrm{H}_{2}$ at 4 torr), growth of well-aligned individual CNTs occurs at a bias voltage of $-550 \mathrm{~V}(360 \mathrm{~W}, 690 \mathrm{~mA})$. In order to achieve the 3 to $5 \mu \mathrm{m}$ length of nanotube that is best for imaging, 10 minutes growth 
time is needed. At certain spots on the cantilever wafer, catalyst sizes of $1 \mu \mathrm{m}$ are patterned as process control sites. Figure 1 shows the SEM images of cantilevers with CNTs grown from 1 $\mu \mathrm{m}$ catalyst sites on a cantilever beam. Normally, more than 20 CNTs can be grown from these 1 $\mu \mathrm{m}$ catalyst spots. During the growth, sometimes the edge CNTs grew faster than the center CNTs. As shown in Figure 1, we were able to get one CNT that protruded 2 to $3 \mu \mathrm{m}$ above the rest of the CNTs. We tested this tip as an AFM imaging probe, and were able to get some good images from this protruded CNT.

We found that with catalyst feature sizes between $200 \mathrm{~nm}$ and $300 \mathrm{~nm}$, we typically obtained 2 to 4 CNTs per site. While with catalyst feature sizes between $75 \mathrm{~nm}$ and $200 \mathrm{~nm}$, only one CNT was grown per site. Figure 2 shows the individual CNT grown from a $100 \mathrm{~nm}$ catalyst site on a cantilever beam which we achieved using our optimized growth conditions. Single CNT growth on cantilevers was very challenging; the growth conditions needed to be controlled very precisely. After a series of systematic study, we finally were able to master the critical conditions for achieving single CNT growth on cantilever structures. This growth breakthrough convinced us as-grown CNTs for AFM tips were in fact feasible.
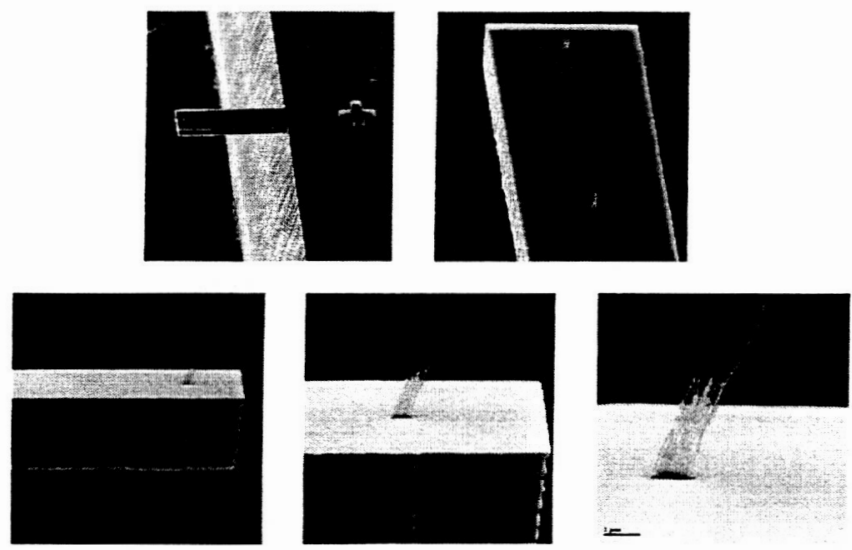

Figure 1. Scanning electron microscopy images of cantilevers with CNTs grown from $1 \mu \mathrm{m}$ catalyst sites on a cantilever beam.
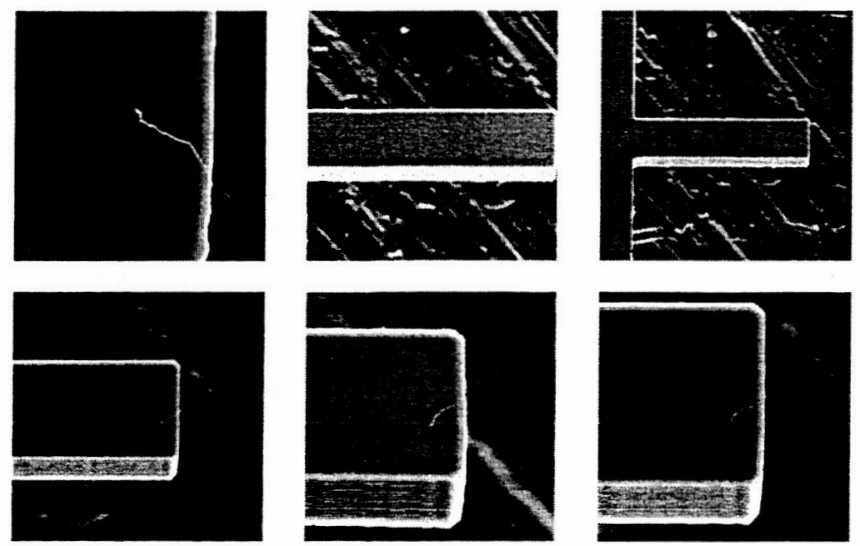

Figure 2. Scanning electron microscopy images of cantilevers with CNTs grown from $100 \mathrm{~nm}$ catalyst sites on a cantilever beam. 

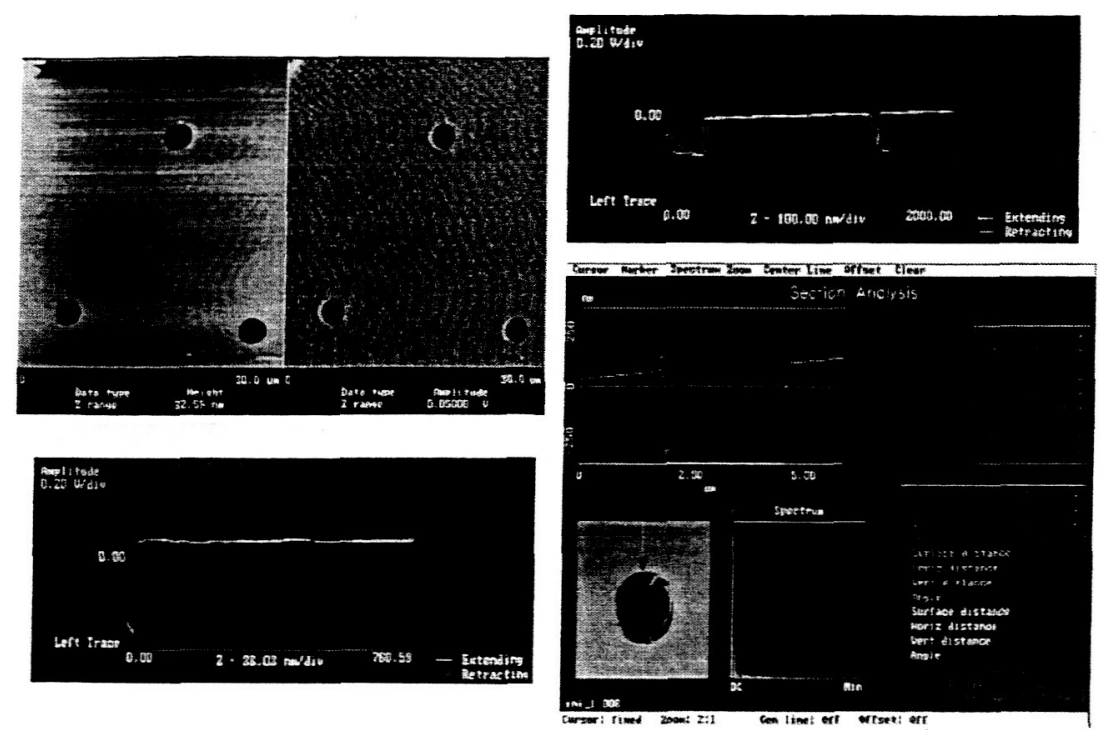

Figure 3. AFM images of an array of $2 \mu \mathrm{m}$ circular holes that are $300 \mathrm{~nm}$ deep using a CNT AFM probe that we made from our wafer-scale fabrication process.

Further growth optimization centered on controlling the uniformity, length, and diameter of the CNTs from the nano-sized catalyst islands over 4" wafer scale. Detailed analysis of the CNT lengths showed a distribution of $+/-20 \%$. It is well understood that the size of the catalyst particle determined the diameter of the CNT. The CNT diameter distributions from $100 \mathrm{~nm}$ catalyst sites were about 50 to $100 \mathrm{~nm}$. Careful inspection of cantilevers on different areas of the wafer allowed us to record statistics of process yield and quality. Due to the 4 " electrode temperature uniformity issue in our PECVD reactor, currently, our wafer scale growth yield was about $10 \%$.

Figure 3 shows AFM images of an array of $2 \mu \mathrm{m}$ circular holes that are $300 \mathrm{~nm}$ deep using a CNT AFM probe that we made from our wafer-scale fabrication process. The force vs. distance curve offered an in-situ technique to examine the mechanical characteristics of the CNT probe tips. The CNT AFM probe that we fabricated exhibited well-defined buckling signatures that resulted from the AFM cantilever pushing towards the sample surface. The fluctuation in the amplitude vs. distance signal indicated that a CNT was present. We also used a validation test to determine the number of tip engagements that the CNT could undergo. We were able to obtain more than 100 tip engagement cycles under ambient imaging conditions. This met our initial goal for assessing CNT probe tip viability. We also compared the imaging lifetime and resolution of conventional silicon tips with our CNT tips by collecting time lapse images of a relatively hard surface composed of a thin layer of silicon nitride. After continuous scanning for more than 8 hours, the CNT probe tip exhibited no detectable degradation in the lateral resolution of the silicon nitride grains, with no loss in apparent grain size $(8-10 \mathrm{~nm})$. The conventional silicon probe tip initially had somewhat poorer resolution when imaging the same surface (12-15 nm grain size), but after one hour of continuous imaging, the tip began to wear, thus the image resolution degraded. This was a clear demonstration of the enhanced performance of CNT-based tips for characterizing surfaces that could cause tip damage or wear. 
The image obtained by the conventional tip was limited by its tip geometry. The silicon pyramid was physically unable to accurately trace the topographic variations of the surface, whereas the CNT-based tip easily reproduced the surface image. Our carbon nanotube tips were able to fully trace the bottom of $1 \mu \mathrm{m}$ trenches without producing image artifacts.

\section{CONCLUSIONS}

For the first time in nanoscience and nanotechnology, this study demonstrated the successful integration of wafer-scale nanopatterning with traditional silicon microfabrication technologies for fabricating carbon nanotube AFM cantilever probes on whole wafers. Our catalyst protection schemes have been proven to be very effective. Plasma enhanced chemical vapor deposition method is utilized for controlling well-aligned carbon nanotube growth. We have successfully developed an innovative nanofabrication process that enabled the batch fabrication of carbon nanotube probe tips (244 tips per 4" wafer) with controlled length, diameter, vertical orientation, and crystalline morphology. Preliminary CNT scanning probe test results show decent buckling effects of the CNTs. This pioneering work sets an excellent example by combining nanomaterials (CNTs) with micron scale devices (cantilevers) for fabricating nanodevices and nanosensors at large quantities.

\section{ACKNOWLEDGMENTS}

The authors would like to thank Mr. Ramsey Stevens for assisting CNT probe testing. This material is based upon work supported by the National Science Foundation under Grant No. 0320512. This work is also supported under NASA contracts (No. NAS2-99092 and No. NAS203144). Integrated Nanosystems Inc. is acknowledged for supplying the PECVD system for this study.

\section{REFERENCES}

1. M.S. Dresselhaus, G. Dresselhaus, Ph. Avouris (Eds.), Carbon Nanotubes, Synthesis, Structure, Properties, and Applications, Topics Appl. Phys. 80, Springer, (2001).

2. J. Li, Y. Lu, Q. Ye, M. Cinke, J. Han, and M. Meyyappan. Carbon nanotube sensors for gas and organic vapor detection. Nano Letters, Vol. 3, No. 7, 929-933 (2003).

3. J. Li, Q. Ye, A. Cassell, H. T. Ng, R. Stevens, J. Han, and M. Meyyappan. Bottom-up approach for carbon nanotube interconnects. Applied Physics Letters, Vol. 82, No. 15, 2491 2493 (2003).

4. A. M. Cassell, Q. Ye, B. A. Cruden, J. Li, P. C. Sarrazin, H. T. Ng, J. Han, and M. Meyyappan. Combinatorial Chips For Optimizing The Growth and Integration of Carbon Nanofiber Based Devices. Nanotechnology. 15, 9-15 (2004).

5. J. Li, H. T. Ng, A. Cassell, W. Fan, H. Chen, Q. Ye, J. Koehne, J. Han, and M. Meyyappan. Carbon nanotube nanoelectrode array for ultra sensitive DNA detection. Nano Letters, Vol. 3, No. 5, 597-602 (2003).

6. B. A. Cruden, A. M. Cassell, Q. Ye, and M. Meyyappan. Reactor design considerations in the hot filament/direct current plasma synthesis of carbon nanofibers. Journal of Applied Physics, Vol. 94, No. 6, 4070-4078 (2003). 